\title{
Documentos para una historia familiar: la familia Trujillo Lanuza (1758-1870)
}

\author{
GUADALUPE CARRASCO GONZALEZ \\ ELISA ROVIRA
}

Durante cinco meses, con financiación de la Institución Cultural "el Broncense" de la Exma. Diputación Provincial de Cáceres, y dentro de la campaña de ordenación de los fondos del Archivo del Real Monasterio de Guadalupe, se realizaron trabajos de expurgo, clasificación e inventario de éstos, bajo la dirección de Fray Sebastián García y Elisa Rovira.

Los fondos inventariados llegaron al Monasterio desde el convento franciscano de la Inmaculada del Palancar, a donde fueron trasladados desde el convento franciscano de San Antonio de Cáceres. En este último edificio se encontraban por haber servido como vivienda particular y depósito de la Delegación de Hacienda durante los años inmediatos a la exclaustración de 1835 .

Los cajones llegados incluían documentación perteneciente a distintas generaciones de la familia materna de D. Tomás Trujillo Lanuza, último eslabón generacional documentalmente conocido y que da nombre al fondo.

El criterio de clasificación seguido en la ordenación de este fondo ha sido sencillo, limitándonos sólo a separar los documentos considerando diferentes conceptos:

A. Documentos considerados como familiares,

B. Documentos cuya génesis está determinada por la actividad profesional de algunos de los miembros de la familia, y

C. Documentación suelta, sin relación aparente con la anterior, que se ha clasificado como Varia. 


\section{A. DOCUMENTACION FAMHIAR MATERNA}

Bajo este epígrafe se han clasificado cinco legajos que comprenden numerosos documentos agrupados en torno a diferentes pleitos entablados por la familia Baraibar. Estos documentos, en su mayoría traslados de los originales, tienen gran valor e interés para aquellos investigadores que se interesen por la "historia familiar", en cuanto que se trata de contratos sucesivos de dote, inventarios de bienes, testamentos, inventarios pos-morten, divisiones de bienes, así como relaciones de las propiedades que cada uno de los cónyuges aportaba al matrimonio. Se ofrece así la posibilidad de aproximarse, en cierta medida, a la realidad de una familia burguesa de la época, a través del estudio de la evolución y estructura de su capital familiar.

Especial mención merecen las relaciones de propiedades rústicas y urbanas que se elaboran -a veces- al contraer matrimonio algunos miembros de la familia, y que son un buen indicador del tipo de estrategia matrimonial seguida y de las preferencias inversoras de sucesivos miembros de la familia.

\section{B. DOCUMENTACION PROFESIONAL}

Un grupo de quince legajos recogen la documentación de carácter profesional perteneciente a D. Florencio de Baraibar, como administrador de la Hacienda que el duque de Abrantes poseía en Cáceres (1); a D. José María Trujillo Cabezas como habilitado del clero de la provincia de Cáceres y a D. Tomás Trujillo Lanuza como procurador de tribunales. Pero sin duda, de todos ellos, los seis legajos que contienen la documentación de la-administración de la Hacienda del duque son los que ofrecen mayor interés.

Contienen estos papeles referencias a algunos de sus mayorazgos (mayorazgo de los Carvajal, Mayorazgo Pizarro-Perero y el de Espadero-Saavedra), fundación de obras pías y capellanías realizadas por el duque y pleitos sobre diferentes cuestiones, especialmente sobre ganados mostrencos con la villa de Montánchez (1793) y con el marqués de la Isla sobre deslindamiento de tierras (1806-1847).

Además el legajo 10 agrupa extractos de cuentas de la administración, un libro de entrada y salida de frutos de la Hacienda del duque

(1) Se incluye una descripción del linaje, títulos, señoríos, vínculos y mayorazgos del duque de Abrantes al comienzo del apartado dedicado a la documentación profesional en la página 16. 
y varios libros de caja de diferentes haciendas situadas en Toledo y Cáceres.

Los legajos 12 al 16 contienen documentación profesional referente a la habilitación del clero. Comprenden, además de numerosos papeles sobre presupuestos, correspondencia y relaciones de las Diócesis de Avila, Ciudad Rodrigo, Coria, Plasencia, Arzobispado de Toledo y Obispado-priorato de San Marcos de León con el Obispado de Cáceres entre 1867-1872, así como documentación sobre algunas obras pías.

Los legajos 17 al 20 incluyen los documentos producidos en su actividad profesional, como procurador de tribunales por $\mathrm{D}$. Tomás Trujillo Lanuza. Estos son : libros de cargo y data, libros de aceptación de poderes, libros de conocimientos de procurador, de notificaciones, de correspondencia y de relación de causas entre 1859 y 1887.

\section{DOCUMENTACION VARIA}

El resto de la documentación se ha clasificado en el legajo 21 bajo el epígrafe de Varia, ante la imposibilidad de establecer su pertenencia con precisión. Tal vez algunos de los documentos puedan tener relación con la administración de la Hacienda del duque de Abrantes, en tanto en cuanto el periodo cronológico que abarca se corresponde con el periodo de tiempo en que fue administrador don Florencio de Baraibar (1785-1811).

Ignoramos también cómo llegaron a parar a este fondo familiar y profesional documentos procedentes del Ayuntamiento de Alcuescar como las diligencias formadas de oficio sobre la construcción de un cementerio (1807) o algunos de los libros de acuerdos municipales de los años 1808-1827. 
FAMIIA MATERNA DE D. TOMAS TRUJHLO LANUZA
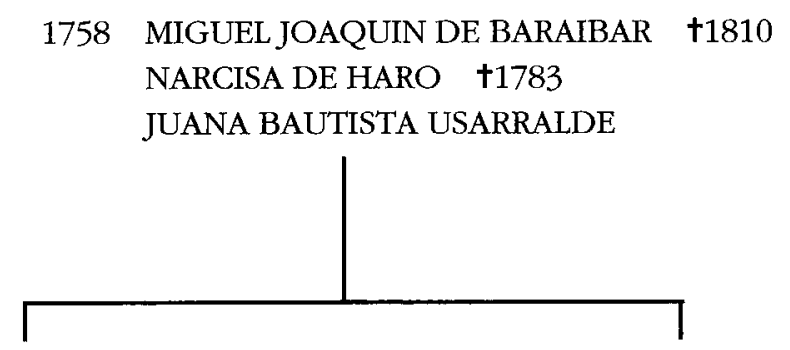

1783 MARTIN P. BARAIBAR Y HARO $\$ 1788$ JOSE F. BARAIBAR Y HARO FRANCISCA MODET

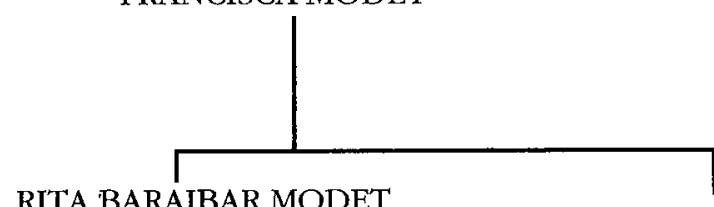

1811 FLORENCIO BARAIBAR MODET MARIA JOSEFA IBARRA $\mathbf{\dagger} 1814$

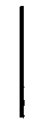

? DOMINICA BARAIBAR IBARRA TOMAS LEANDRO DE LANUZA

$$
1
$$

TOMAS TRUJILLO LANUZA 


\section{A. DOCUMENTACION FAMILIAR MATERNA \\ (Legajos 1-5)}

\section{LEGAJO 1: M. JOAQUIN DE BARAIBAR-NARCISA DE HARO}

Carp. 1. Pleito entre D. M. Joaquin de Baraibar y sus hijos sobre los bienes de $D^{a}$ Narcisa de Haro (1758-1832)

- Traslado autorizado del contrato matrimonial otorgado en 1758 entre D. Miguel Joaquín de Baráibar y Ochotorena y $\mathrm{D}^{\mathrm{a}}$ Narcisa de Haro (Estellla, 1783).

- Traslado autorizado del testamento de D Narcisa de Haro (Estella, 1783).

- Inventario de los bienes que quedaron en casa de D. M. Joaquín de Baráibar a la muerte de su esposa, D ${ }^{a}$ Narcisa de Haro (Estella, 1783).

-Adjunta copia"simple.

- Traslado autorizado del contrato matrimonial otorgado entre D. Martín Prudencio de Baráibar y. Haro y D Francisca Modet (Estella, Junio de 1783).

-Adjunta en el mismo documento copia de la carta de pago de la dote de la desposada.

(Existe otra copia autorizada en el leg. 2)

- Copia simple del inventario de los bienes entregados a D. Martín Prudencio de Baráibar por su padre, al contraer matrimonio con $\mathrm{D}^{\mathbf{a}}$ Francisca Modet (Estella, 1783).

- Papeles varios pertenecientes a D. Manuel Modet, en su calidad de curador y suegro del menor D. Martín P. de Baráibar (1783-88).

- Testamento de D. José Felipe de Baráibar y Haro (Estella, 1784).

- Auto de revocación de curaduría y nuevo nombramiento de curador de los intereses de D. José Felipe de Baráibar a favor de D. Manuel Pastor (1785).

- Escritura pública de convenio, otorgada entre los hermanos Martín Prudencio y José Felipe de Baráibar en razón de los posibles intentos paternos para desheredarles (Olazagutia, 1785).

-Adjunta tres copias simples de la obligación privada previa.

- Traslado autorizado del testamento de D. M. Joaquín de Baráibar (Estella, 1796)

- Idem del codicilo (Estella, 1803).

- Inventario de los bienes recibidos por $\mathrm{D}^{\mathrm{a}} \mathrm{Juana}$ Bautista de Usarralde, segunda esposa de D. M. Joaquín de Baráibar y Ochotorena, a la muerte de éste (Estella, 1810). 
- Escritura de reparto de bienes, otorgada entre $D^{a}$ Juana Bautista de Usurralde y los demás herederos de D. M. Joaquín de Baráibar (Estella, 1814)

- Traslado autorizado de la escritura de convenio otorgada en 1831 entre $\mathrm{D}^{\underline{a}}$ Juana Bautista de Usarralde y D. Manuel Modet, abuelo y curador de los menores D. Florencio y $\mathrm{D}^{\mathrm{a}}$ Rita de Baráibar y Modet (Estella, 5-XI-1831).

-Adjunta carta de pago (Estella, 29-II-1832).

- Correspondencia varia sobre el pleito (1783-1831)

\section{Carp. 2. Pleito contra D. M. Joaquín de Baráibar.}

- Copia autorizada de los autos del pleito resuelto a favor de D. M. Joaquín de Baráibar y Ochotorena, sobre el cargo interpuesto contra él por supuesto uso indebido de blasones (Pamplona, 1772.)

\section{LEGAJO 2: MARTIN P. DE BARAFBAR Y HARO-FRANCISCA MODET}

Carp. 1. Traslado autorizado del contrato matimonial otorgado entre D. Martín Prudencio de Bráibar y Haro y $\mathrm{D}^{\mathbf{a}}$ Francisca Modet, en unión de sus padres respectivos (Estella, 1783).

-Adjunta carta de pago de la dote de la novia en el mismo documento.

(Véase leg. 1, cap.1).

\section{Carp. 2. Testamentaría de $D$. Martín P. de Baráibar.}

- Traslado autorizado del testamento otorgado por D. Martín Prudencio de Baráibar y Haro en 1784 (Tafalla,1788)

- Idem del nuevo testamento otorgado en 1788 , poco antes de su muerte (Estella, 1788).

Carp. 3. Relación duplicada y recibos de los gastos ocasionados por el entierro de D. Martín P. de Baráibar (1778).

Carp. 4. Traslado autorizado del inventario de los bienes recibidos en 1788 por $\mathrm{D}^{\mathrm{a}}$ Francisca Modet, a la muerte de su esposo D. Martín P. de Baráibar y Haro (Estella, 1796).

Carp. 5. Traslado autorizado de la carta de pago otorgada por $\mathrm{D}^{\mathrm{a}}$ Francisca Modet a favor de su padre, D. Manuel Modet, al finalizar la actividad de éste como curador de los intereses de $\mathrm{D}^{\mathbf{a}}$ Francisca (Estella, 1796). 
-Adjunta dos relaciones de honorarios del escribano (1796) (Tanto estos documentos como los de la carpeta anterior se emitieron con motivo del nuevo matrimonio contraído por Dª Francisca Modet con D. José Bodet en 1796, motivo por el cual cesó la actividad de D. Manuel Modet como curador de su hija, quedando al tanto de los intereses de sus nietos, D. Florencio y $\mathrm{D}^{\underline{a}}$ Rita de Baráibar, en todo aquello referido a la herencia paterna de ambos menores)

\section{Propiedades del matrimonio Baráibar-Modet}

Carp. 6. Traslado autorizado de la documentación relativa a la venta de una viña, realizada por Josefa de Senosiaín, viuda de José Galdeano, a favor de D: Martín P. de Baráibar (Estella, 1784).

\section{Carp. 7. Adquisiciones realizadas a $\mathrm{D}^{\mathrm{a}} \mathrm{M}^{\mathrm{a}}$ Jerónima de Oteiza}

A) - Escritura pública de compra-venta de casa y varias fincas con cargo de censo, otorgada por $\mathrm{D}^{\underline{a}} \mathrm{M}^{\mathrm{a}}$ Jerónima de Oteiza a favor de D. Martín P. de Baráibar y su esposa (Estella, 1785).

- Traslado autorizado del auto de depósito de 150 ducados, efectuado por D. Martín P. de Baráibar y D ${ }^{\underline{a}}$ Francisca Modet como redención del censo impuesto a favor de la capellanía fundada por D. Francisco de Benavides sobre parte de los bienes adquiridos a $\mathrm{D}^{\mathrm{a}} \mathrm{M}^{\mathrm{a}}$ Jerónima de Oteiza (Estella, 1785).

- Idem del auto de depósito de 50 ducados, efectuado para redimir el censo impuesto, sobre otra parte de los bienes adquiridos a la Sra. Oteiza, a favor de la obra pía instituida por $D^{\mathbf{a}}$ Juana de Dicastillo (Estella, 1785).

-Adjunta carta de pago.

B) Escrituras antiguas de las propiedades adquiridas a $D^{\underline{a}} M^{a}$ Jerónima de Oteiza (1601-1785):

- Escritura pública de remate de pago de la parte de la viña de Aiztondeta, adquirida por Juan de Oteiza a María de Soles en 1600 (Estella, 1601).

- Traslado autorizado de la información realizada con motivo de la petición que hizo $\mathrm{D}^{a}$ Isabel Díaz de Allo, viuda de D. Diego de Oteiza, para descepar una viña de su propiedad, sita en San Martín de Aldur (Estella, 1646).

- Copia simple de la documentación de compra-venta de una casa en la ciudad de Estella, otorgada por D. Pedro de Echávarri, vecino de Los Arcos, a favor de D. Bernardo de Oteiza, vecino de Estella (Estella, 1661). 
- Traslado autorizado de la escritura de compra-venta de una viña, otorgada por D. Francisco Bernardo de Oteiza, como apoderado de $\mathrm{D}^{\mathbf{a}}$ Laurencia de Cruzate y Zúñiga, a favor de D. Francisco de Oteiza, su sobrino (Estella, 1682).

-Adjunta:

- Dos poderes otorgados por $\mathrm{D}^{\mathrm{a}}$ Laurencia de Cruzate y Zúñiga a favor de D. Francisco Bernardo de Oteiza (Puerto de Santa María, 1681).

- Poder otorgado por D. Bernardo de Cruzate y Zúñiga a favor de José de Oteiza (Puerto de Santa María, 1669)

- Copia autorizada del testamento otorgado en 1667 por $\mathrm{D}^{\mathrm{a}}$ Laurencia y D. Bernardo de Cruzate y Zúñiga (Puerto de Santa María, 1699).

- Traslado autorizado de la escritura de tasación y venta de una viña con olivos, otorgada por Matías de Solórzamo y su mujer a favor de D. Miguel Jerónimo de Oteiza (Estella, 1717).

- Escritura de licencia otorgada a favor de D. Miguel Jerónimo de Oteiza para roturar cuatro peonadas de una viña vieja de su propiedad (Estella, 1730).

- Testamento de hermandad otorgado por doña Juana $M^{a}$ y doña $M^{\underline{a}}$ Santos de Oteiza (Estella, 1724).

- Testamento otorgado por $\mathrm{D}^{\mathrm{a}} \mathrm{M}^{\mathrm{a}}$ Santos de Oteiza (Estella, 1743).

- Escritura de reconocimiento de censo y carta de pago del valor de una heredad, otorgada, en virtud de facultad del alcalde de la ciudad de Estella, por Juan Bernardo de Arteaga y consortes a favor de $\mathrm{D}^{\underline{\underline{a}}} \mathrm{M}^{\underline{a}}$ Jerónima de Oteiza (Estella, 1785).

Carp. 8. Escritura de obligación de pago de un préstamo otorgada por Norberta de Puyarena a favor de D. Martín P. de Barbáibar (Estella, 1787).

Carp. 9. Escritura de arrendamiento de una casa sita en la ciudad de Estella, otorgada por $D^{\underline{a}}$ Francisca Modet a favor de D. Pantaleón Lorente y su mujer (Estella,1794).

\section{Carp. 10 Escritura de las propiedades procedentes de la familia Modet (1734-1757).}

- Escritura de compra-venta de una viña, otorgada por José de Mondragón y consorte a favor de Domingo de Vicuña (Estella, 1743).

- Adjunta copia simple.

- Escritura de conpra-venta de una viña, otorgada por D. Antonio Modet a favor de D. Domingode Vicuña (Estella, 1747). 
- Escritura de imposición de censo, otorgada por José de Arizaleta y su mujer a favor de Da Paula Modet (Estella, 1751).

- Escritura de compra-venta de una viña, gravada con censo, otorgada por Dá Paula Modet, viuda de D. Domingo de Vicuña, a favor de José de Arizaleta (Éstella, 1757).

\section{LEGAJO 3: FLORENCIO DE BARAIBAR Y MODET-M." JOSEFA DE IBARRA Y LIZARANZU}

Carp. 1. Traslado autorizado de las escrituras de institución de curaduría para los menores $\mathrm{D}^{\mathrm{a}}$ Rita y D. Florencio de Baráibar y Modet, discernida a favor de su abuelo, D. Manuel Modet (Estella, 1788).

Carp. 2. Escritura del auto de entrega de los bienes que quedaron a la muerte de D. Martín P. de Baráibar, otorgada por $\mathrm{D}^{\mathbf{a}}$ Francisca Modet a favor de su padre, D. Manuel Modet, como autor y curador de D. Florencio y $\mathrm{D}^{\mathbf{a}}$ Rita de Baráibar, sus hijos (Estella, 1796).

Carp. 3. Correspondencia de D. Felipe de Baráibar con D. Manuel Modet, sobre deudas contraídas con sus sobrinos, D ${ }^{\mathbf{a}}$ Rita y D. Florencio de Baráibar (1799).

(4 documentos).

Carp. 4. Redención de censos impuestos sobre los bienes adquiridos a $\mathrm{D}^{\mathrm{a}} \mathrm{M}^{\mathrm{a}}$. Jerónima de Oteiza (1807).

- Escritura de redención del censo impuesto a favor de la capellanía fundada por D. Francisco Bernardino de Oteiza. Otorgada a favor de D. Manuel Modet, como tutor y curador de su nieto D. Florencio de Baráibar.

- Adjunta la escritura de imposición del censo (Estella, 1695).

- Escritura de redención del censo impuesto a favor del Mayorazgo de Tarazona. Otorgada a favor de D. Manuel Modet, como tutor y curador de D. Florencio de Baráibar.

Carp. 5. Escritura de obligación de pago de un préstamo, otorgada por D. Miguel Cabrera, por sí y como apoderado de $D^{\mathbf{a}}$ Casilda y D. Manuel de Larramendi, a favor de D. Florencio de Baráibar (Estella, 1811).

-Adjunta testimonio firmado de recepción del préstamo. 
Carp. 6. Papeles sobre la tasación de la casa y heredades de D. Florencio de Baráibar en la ciudad de Estella (1812-13).

(4 documentos).

Carp. 7. Préstamo realizado por D. Miguel Ramón Modet a D. Florencio de Baráibar y Modet.

- Copia simple de la escritura pública de préstamo otorgada por D. Miguel Ramón Modet, Ministro del Consejo Real, a favor de su sobrino, D. Florencio de Baráibar, para pago de la fianza exigida para acceder al cargo de administrador de la hacienda del Duque de Abrantes en Cáceres. (Original expedida en Madrid, ante D. Juan Raya, notario, el 1-VII-1828).

- Escritura pública de pago, otorgada entre los herederos de D. Miguel Ramón Modet y $\mathrm{D}^{\mathrm{a}} \mathrm{M}^{\mathrm{a}}$ Dominica de Baráibar e Ibarra, hija y heredera de D. Florencio de Baráibar, ante D. Justo de Sancha, notario (Madrid, 19-II-1847).

Carp. 8. Libro número uno de D. Martín de Baráibar para forasteros (1784-1811).

21h; 585 págs.; 29x20cm. Enc. Pergamino

Contiene realmente dos libros distintos:

- Cuentas de D. Martín de Baráibar (1784-87) (págs. 1-130).

- Relación y cuentas de todos los documentos y bienes pertenecientes a D. Florencio y D. ${ }^{\text {a }}$ Rita de Baráibar, administrados por su abuelo y curador, D. Manuel Modet (1788-1811) (págs. 131-134).

Carp. 9. Documentos personales de $D^{\mathbf{a}}$ Josefa de Ibarra.

- Carta de esclavitud de la Congregación de los Esclavos del Santísimo Sacramento del Caballero de Gracia (Madrid, 22-VI-1801)

(Se trata de un pliego impreso, con un grabado sobre plancha de cobre firmado por M.S. Carmona en 1787).

LEGAJO 4: FLORENCIO DE BARAIBAR Y MODET-M. ${ }^{\text {a }}$ JOSEFA DE IBARRA Y LZARANZU (II)

PLEITO CONTRA EL VIZCONDE DE SANTO DOMINGO (1826-1846)

\section{Carp. 1. Precedentes del pleito (1813-1826).}

- Borrador de la sentencia de arbitrio privado, dada en el litigio habido entre $D^{\mathbf{a}}$ Martina Ignacia de Lizaranzu y Loinaz y D. Domingo José de Ibarra y Lizaranzu, su hijo, sobre las dotes y alimentos debidos por el Vizconde a su madre y hermanas (Lequeitio, 3-VIII-1813). 
- Traslado autorizado de la escritura de transacción otorgada en 1813 entre D. Domingo José de Ibarra y su madre, $D^{\mathrm{a}}$ Martina Ignacia de Lizaranzu y Loinaz (Marquina, 1826).

- Copia simple de la sentencia dictada en Ceanuri sobre el pleito sostenido entre D. Domingo José de Ibarra y Lizaranzu, Da Martina Ignacia de Lizaranzu y Loinaz y los acreedores surgidos a la muerte de D. Domingo José de Ibarra y Lizaranzu, padre, anterior Vizconde de Santo Domingo (Original dada en Ceanuri, 11-VIII-1826).

\section{Carp. 2. Autos y ejecutorias (1829- ¿1840?).}

- Notificación de autos, dirigida a D. Domingo José de Ibarra en razón del pleito seguido contra él por $\mathrm{D}$. Florencio de Baráibar en representación de su hija, $\mathrm{D}^{\mathrm{a}} \mathrm{M}^{\mathrm{a}}$ Dominica de Baráibar e Ibarra, sobre la dote de $\mathrm{D}^{\mathbf{a}} \mathrm{M}^{\mathrm{a}}$ Josefa de Ibarra y Lizaranzu (Tolosa, 1829).

- Copia simple de la real ejecutoria obtenida en la Cancillería de Valladolid por $D$. Florencio de Baráibar, como padre, tutor y curador de $D^{a} M^{a}$ Dominicana de Baráibar e Ibarra, en el pleito contra el Vizconde de Santo Domingo (Original dada en Valladolid el año 1832).

- Copia simple del expediente ejecutorio incoado por D. Florencio de Baráibar contra el Vizconde de Santo Domingo, sobre el cumplimiento de la ejecutoria expedida por la Cancellería de Valladolid (Original: Valladolid, Julio, 1832).

- Copia simple de la real provisión de la Cancillería de Valladolid, expedida en Marzo de 1834 sobre el pleito, aún no resuelto.

- Copia simple de la memoria y petición formuladas por D. Vicente de Azpiazu e Iturbe, como apoderado de D. Tomás Leandro de Lanuza, marido y administrador de D..$^{\underline{a}}$ M. ${ }^{\underline{a}}$ Dominica de Baráibar e Ibarra, en razón del pleito pendiente contra D. Domingo José de Ibarra (Original: Madrid, ¿1840?).

\section{Carp. 3. Documentos aportados al pleito como testificaciones.}

- Escritos matrimoniales de D. Florencio de Baráibar y $D^{\underline{a}} M^{a}$ Josefa de Ibarra y Lizaranzu (Pamplona, 1811).

- Traslado autorizado del contrato matrimonial otorgado en 1811 entre D. Florencio de Baráibar y $\mathrm{D}^{\underline{a}}$ y $\mathrm{M}^{\mathrm{a}}$ Josefa de Ibarra y Lizaranzu (Pamplona, 1811).

- Traslado autorizado y legalizado del acta de matrimonio de D. Florencio de Baráibar y $\mathrm{D}^{\mathrm{a}} \mathrm{M}^{\mathrm{a}}$ Josefa de Ibarra, fechada el 15-IX1811. (Estella, 1811).

- Traslado autorizado del testamento de $\mathrm{D}^{\mathrm{a}} \mathrm{M}^{\mathrm{a}}$ Josefa de Ibarra y Lizaranzu, otorgado en 1814 (Estella, 1826). 
- Documentación de $\mathrm{D}^{\mathbf{a}}$ Martina Ignacia de Lizaranzu y D. Domingo J. de Ibarra y Lizaranzu, mayor, padres de $\mathrm{D}^{\mathrm{a}} \mathrm{M}^{\mathrm{a}} \mathrm{J}$ Josefa y $\mathrm{D}$. Domingo J. de Ibarra:

- Copia simple del contrato matrimonial otorgado en la anteiglesia de San Pedro de Berriatúa, señorío de Vizcaya, entre D. Domingo José de Ibarra y Lizaranzu y $\mathrm{D}^{\mathbf{a}}$ Martina Ignacia de Lizaranzu y Loinaz en 1790.

- Copia simple de los autos resueltos en 1716 sobre la participación de los bienes dejados en herencia por D. Martín Benito de Loinaz y $\mathrm{D}^{\mathrm{a}}$ Isabel $\mathrm{M}^{\mathrm{a}}$ de Bustamante, Marquesa de Gracia Real, su hija, y a $D^{\mathrm{a}}$ Martina de Lizaranzu y Loinaz, su nieta.

(Faltan algunos folios al principio).

- Traslados autorizado del testamento otorgado en 1807 por D. Domingo J. de Ibarra (Marquina, 1817).

- Condiciones de necesario cumplimiento por parte de $\mathrm{D}^{\mathrm{a}}$ Martina Ignacia de Lizaranzu y Loinaz para el otorgamiento de la escritura de capitulaciones matrimoniales entre D. Domingo J. de Ibarra, su hijo, y D. ${ }^{\text {F }}$ Francisca de Ayala. (Sin fechas)

(Dos copias).

- Copia simple del testamento otorgado en 1819 por $\mathrm{D}^{\mathrm{a}}$ Martina Ignacia de Lizaranzu y Loinaz y del inventario de los bienes que dejó a su muerte, acaecida en 1843.

- Propiedades del Vizconde de Santo Domingo:

- Relación de los bienes correspondientes a los mayorazgos del Vizconde de Santo Domingo.

- Papeles varios relativos a las propiedades inmuebles del Vizconde de Santo Domingo en Madrid.

Carp. 4. Correspondencia sobre el pleito (1817; 1826-29; 1844-46).

LEGAJO 5: TOMAS L. LANUZA-Mํㅡㅁ DOMINICA BARAIBAR E IBARRA PROPIEDADES Y RENTAS

Carp. 1. Títulos de propiedad de la dehesa "Valhondo de Holguines", adquirida por $D$. Tomás Leandro de Lanuza y heredada por su hija, $D^{\underline{a}}$ Maltide Lanuza y Baráibar.

- Escritura pública de compra-venta de 125 maravedís de renta de hierbas, otorgada por D. Víctor Izquierdo Pizarro a favor de D. Tomás Leandro de Lanuza ante el escribano D. Diego Ladrón de Guevara (Cáceres, 13-VIII-1847). 
- Escritura pública de compra-venta de 19 maravedís y 9/12 de renta de hierbas, otorgadas por D. Jacinto de Orellana Pizarro, Marqués de la Conquista, a favor de D. Tomás Leandro de Lanuza ante el escribano D. Julián Ramos Blanco (Trujillo, 10-III-1848).

- Escritura pública de compra-venta de 53 maravedís y $1 / 12$ de renta de hierbas, otorgada por D. Manuel García del Prado, $D^{\mathbf{a}}$ Rafaela y $\mathrm{D}^{\mathrm{a}}$ Josefa Michel a favor de D. Tomás Leandro de Lanuza ante el escribano D. Lorenzo Mendoza (Cáceres, 5-XI-1850).

- Escritura pública de compra-venta de 20 maravedís y $5 / 6$ de renta de hierbas, otorgada por D. Fernando García Becerra a favor de D. Tomás Leandro de Lanuza ante el escribano D. Lorenzo Mendoza (Cáceres, 1-VII-1852).

- Escritura pública de venta judicial de 17 maravedís de renta de hierbas a favor del Marqués de Iturbieta, ganadero trashumante, vecino de Madrid. Otorgado por D. Diego de Carvajal Roco de Godoy (Cáceres, 6-IV-1799).

(Esta participación pasó a manos de D. Joaquín Fagoaga, cuyo título de propiedad no se halla entre estos documentos. A Fagoaga se la adqurió D. Tomás Leandro Lanuza en venta judicial. Véase la escritura siguiente).

- Escritura de venta judicial de 358 maravedís de renta de hierbas. Otorgada por los síndicos de la quiebra de D. Joaquín Fagoaga a favor de D. Bernardo Brieva, como apoderado y en nombre de D. Tomás Leandro de Lanuza, ante el Tribunal de Comercio de Madrid (Madrid, 20-V-1856).

- Adjunta:

- Recibos de pago.

- Carta de aceptación de dote, otorgada por la comunidad de monjas clarisas de S. Pablo, de la villa de Cáceres, al recibir como monjas profesas a María de Aldana y Juana Blázquez, hijas de Pedro Alvarez Holguín (Cáceres, 24-V-1515, ante Gaspar Sánchez, escribano).

- Carta de aceptación de dote, otorgada por la comunidad de monja clarisas de S. Pablo al recibir como monjas profesa a Beatriz Alvarez, hija de Francisco de Figueroa y de María Alvarez (Cáceres, 20-II-1520 ante Gaspar Sánchez, escribano público).

(En ambas cartas de dote se incluyen rentas de hierbas situadas sobre la dehesa Valhondo de Holguines).

- Escritura de permuta de 18 maravedís $5 / 6$ y $1 / 3$ de rentas de hierba, otorgada entre el Conde de Adanero y D. Tomás Leandro de Lanuza ante D. Saturnino González Celaya, escribano (Cáceres, 29-IX-1862). 
- Escritura de permuta de 77 marvedies y 1/2 de renta de hierbas, otorgada entre D. Francisco Castor Muñoz y D. Tomás Leandro Lanuza y Molinero ante D. Saturnino González Celaya, escribano (Cáceres, 19-VI-1863).

- Escritura de compra-venta de 75 maravedíes y $1 / 2$ de renta de hierbas, otorgada por D. Alonso Núnez Parrón a favor de D. Tomás Leandro Lanuza y Molinero ante D. Saturnino González Celaya, escribano (Cáceres, 8-X-1870).

- Escritura de permuta de 55 maravedís y $1 / 2$ de renta de hierbas. Otorgado entre D. Tomás Leandro de Lanuza y D. Juan Guerra Carrasco, como apoderado de la Marquesa de Santa Marta, ante D. Saturnino González Celaya (Cáceres, 28-II-1871).

\section{Carp. 2. Otras propiedades rústicas.}

- Documentos sobre arrendamiento de tierras y molinos, sitos en los términos de Cáceres y Plasencia respectivamente (1848-83).

\section{Carp. 3. Propiedades Urbanas.}

- Arrendamientos de casas pertenecientes a la familia Lanuza-Baráibar:

- En Cáceres (1853-83):

Calle de la Audiencia.

$"$ "Moreras.

Plazuela de S. Blas (dos casas).

$$
\text { " S. Juan. }
$$

Calle de Valdés.

$"$ Villalobos.

- En Estella (1843; 1847-48; 180)

\section{Carp. 4. Rentas varias.}

- Relación de rentas cobradas por D. Tomás Leandro de Lanuza (1853-76). 


\section{B. DOCUMENTACION PROFESIONAL}

\section{B.1. D. Florencio Baráibar y D. Tomás L. Lanuza.}

- Administración de la hacienda del Duque de Abrantes en Cáceres.

(Legs. 6-11).

B.2. D. José $M^{\mathbf{a}}$ Trujillo Cabezas.

- Habilitación del clero de la provincia de Cáceres. (Legs. 12-15)

- Otros cargos. (Leg.16).

\section{B.3. D. Tomás Trujillo Lanuza.}

- Procuraduría de Tribunales. (Legs.17-20). 


\section{- B.1. DOCUMENTOS PROCEDENTES DE LA ADMINISTRACION DE LA HACIENDA DEL DUQUE DE ABRAN'TES EN CACERES. (Legs. 6-11)}

ANEXO AL LEGAJO 11: DOCUMENTACION DE LA EXCELENTISIMA DIPUTACION PROVINCIAL DE CACERES.

(Papeles profesionales de D. Tomás Leandro Lanuza).

IINAJE, TITULOS Y MAYORAZGOS DEL DUQUE DE ABRANTES, D. ANGEL $M^{\text {a }}$ FRANCISCO DE CARVAJAL

\section{LINAJE}

Angel Mํaㄹ Francisco de Carvajal, Fernández de Córdoba, Gonzaga, Zúñiga, Lancáster, Enríquez, Fernández Noreña, Sande, Padilla, Enríquez de Anaya, Vívero Peñalosa, Bobadilla, Ramírez de Arellano, Cano Moctezuma, Silva, Toledo, Pizarro Moscoso, Pimentel, Acuña, Nuño de Castro, Ayala y Enjarada, Mejorada, Portalegre y Villalba.

\section{TITULOS Y SEÑNORIOS}

- Duque de Abrantes y Linares.

- Marqués de Valdefuentes, Aguilafuerte, Villalba de los Llanos, Sandoval, Gouveia y Navamorcuende.

- Poseedor de los Cameros, Asín, estados de Matadeón y Andaluz, de las cinco villas y valle de Canales, de la casa y estados de Baza, del de Abarca y el de Villarramiro, de la villa de Arellano, Soto, Luezas, Castroverde y lugares de su jurisdicción, Piñel de Arriba, Sotosalvos y Pelayos, Fuenbellida, de Vallegera, su torre y casa fuerte y de la de Albillos, de las villas de Navamorcuende, Cardiel, Bodón, Almendral, Sartajada, Buenaventura y Sotillo de las Palomas.

- Grande de España de primera clase.

- Maestrante de la Real Maestranza de Sevilla.

- Gentilhombre de Cámara de Su Majestad.

\section{VINCULOS Y MAYORAZGOS}

- Vínculo-mayorazgo instituido en 1509 por Da María de Peñalosa, mujer de D. Francisco de Bobadilla, a favor de su hijo, D. Antonio de Bobadilla, y su descendientes. 
- Vínculo instituido en 1531 por Mencía Alvarez de Toledo, mujer de Juan de Carvajal, a favor de su hijo, Hernando de Carvajal, y sus descendientes.

- Vínculo instituido en 1568 por Juan Pizarro a favor de su hijo, Alonso Pizarro, y sus descedientes.

Agregación realizada en 1577 por Alonso Pizarro Perero.

- Mayorazgo instituido en 1499 por el Obispo de Burgos D. Luis de Acuña, a favor de su hijo legitimado, D. Diego Osorio, y sus descendientes.

- Mayorazgo instituido en 1512 por D. Juan de Sande y su esposa, $\mathrm{D}^{\mathrm{a}}$ Leonor de Saavedra, a favor de su hijo, D. Juan de Sande y Carvajal, y sus sucesores.

Agregaciones realizadas por el Arcediano de Plasencia D. Francisco de Carvajal; en 1546 y 1551.

- Mayorazgo fundado en 1524 por D. Enrique Enríquez y su esposa, Da María de Luna, a favor de su nieto, D. Enrique Enríquez, y sus descendientes.

Agregación efectuada por el mismo. D. Antonio de Acuña y su hermano, D. Hernando, en 1569.

- Mayorazgo fundado en 1571 por Juan Cano a favor de su hijo Pedro Cano, y sus descendientes (2).

\section{LEGAJO 6: MAYORAZGOS DE LOS CARVAJAL}

\section{Carp. 1. Dehesa "Cabezas de Mariagüe".}

- Escrituras públicas relativas a las participaciones de los Carvajal en la dehesa, sita én el término de Monroy, tierra de Plasencia.

(Se trata de una colección de cuarenta y cinco instrumentos públi$\mathrm{cos}$; encuadernados sin un riguroso criterio cronológico).

\section{Carp. 2. Cuentas de la hacienda de Dª Isabel de Carvajal (1591).}

- Original y copia.

(2) Estos datos han sido extraídos de la copia de una real facultad, concedida al Duque de Abrantes, $D$. Angel $M^{a}$ Francisco de Carvajal y Fernández de Córdoba, en 1814, para vender algunos bienes pertenecientes a sus mayorazgos y del traslado del poder otorgado en 1817 por el mismo Duque a favor de su administrador de Cáceres, D. José Rodríguez Hurtado, para gestionar parte de las mencionadas ventas.

(Véase leg. 7. carp 4).

Para obtener más datos sobre la genealogía de la casa de Abrantes, puede consultarse en el fondo histórico del Afrchivo el legajo número 74 y el cuadro genealógico realizado en el siglo XVIII sobre la descendencia de D. Juan Blázquez de Cáceres y D ${ }^{\underline{a}}$ Teresa Alfón. 


\section{Carp. 3. Censos.}

- Escritura de imposición de censo, cargado por D. Diego Esteban de Carvajal y Nieto, Comendador de Castroverde, sobre 83 maravedís de renta de hierbas en dehesa "Cabezas de Mariagüe", a favor del colegio-seminario fundado en la villa de Cáceres por D. García Galarza, Obispo de Coria (Cáceres, 23-VII-1609, ante Juan Romero, escribano público).

- Escritura de testimonio de la redención del censo, expedida por Pedro Pérez, escribano público (Cáceres, 17-VI-1616).

- Nuevo testimonio de redención del censo, expedido por Pedro Pérez (Cáceres, 18-IV-1619).

LEGAJO 7: MAYORAZGOS DE ESPADERO-SAAVEDRA, PIZARRO PERERO Y OTROS.

\section{Carp. 1. Mayorazgos de los Espadero-Saavedra.}

- Vínculo de Alvaro Gil Mogollón:

- Escrituras varias relativas a las partes de la dehesa "Cabezas de Mariagüe" que pertenecieron al vínculo fundado por Alvaro Gil Mogollón, poseído por los Espadero-Saavedra (1624-1878). (10 cuadernillos)

\section{Carp. 2. Mayorazgos de los Espadero-Saavedra.}

- Escrituras antiguas de las tierras heredadas por el Duque de Abrantes en Torrequemada a través de los Espadero-Saavedra (siglos XV-XVIII):

- Escritura pública de compra-venta de dos viñas, otorgada por Olalla García Raposo ante Diego de Llerena, escribano público (Torrequemada, 12-V-1575).

- Escritura pública de compra-venta de dos partes de tierra en el ejido de Torrequemada, otorgada por Rodrigo Alfón a favor de Algón Gil, ante Ferrán Alfón, escribano (Trujillo, 16-XI-1447).

(Este documento va unido a los que se detallarán a continuación y es el más antiguo con que cuenta este fondo).

- Escritura de compra-venta de una parte de cerca, otorgada por Francisco Fernández Polo a favor del vínculo fundado por D. Fr. Jerónimo Espadero de Loaisa (Torremocha, 29-X-1711, ante Juan de Palacios).

- Memoria de la medida de las tierras heredadas de $D^{\mathbf{a}}$ Isabel Macotela (s. XVIII). 
- Escritura pública de compra-venta de 13 fanegas de tierra en el valdío de Torrequemada, otorgada por María González la Valdesa a favor del vínculo fundado por D. Fr. Jerónimo Espadero de Loaisa, poseído por Dª Francisca Petronila Espadero (Cáceres, 5V-1715, ante Juan Sánchez Pozo, escribano).

- Ejecutoria ganada en la Chancillería de Granada por Rodrigo de Ovando Godoy en el pleito sostenido, como marido de $D^{\mathbf{a}}$. Francisca Petronila Espadero, contra $D^{\underline{a}}$ Catalina Pacheco sobre la hacienda de Torrequemada (Granada, 1-VII-1737).

- Escritura pública de compra-venta de 10 fanegas de tierra en Torrequemada, otorgada por los religiosos del convento de Santo Domingo de la villa de Cáceres a favor de Antón Hernández, ante Manuel Barrio del Pino, escribano (Cáceres, 10-III-1689).

-Adjunta escritura de testimonio de toma de posesión judicial de las tierras por parte de Antón Hernández, ejecutada el 5-V-1689 ante el escribano Manuel Barrio del Pino.

- Escritura pública de testimonio del concurso de acreedores y de adjudicación de las tierras que quedadon a la muerte de D. Francisco de Carvajal y Sande a favor del convento de religiosos de Santo Domingo, de la villa de Cáceres. Expedida por Manuel Barrio del Pino (Cáceres, 12-III-1689).

- Escritura pública de compra-venta de 9 fanegas de tierra, otorgada por el Consejo, Justicia y Regimiento de Torrequemada a favor del vínculo instituido por D. Fr. Jerónimo Espadero, como medio para obtener fondos con que subvenir a las necesidades de la guerra (Torrequemada, 29-I-1712), ante Juan Sánchez Pozo, escribano).

- Cuentas varias de los bienes dejados en herencia por $\mathrm{D}^{\mathrm{a}}$ Isabel Macotela en Torrequemada (1730-36).

- Petición formulada por Francisco de Córdoba en nombre de Francisco Gutiérrez, curador de Da Francisca Petronila Espadero, para la tasación de las tierras de Torrequemada (1703).

- Memoria de las tierras de Torrequemada (1668).

(2 hojas sueltas, muy deterioradas).

\section{Carp. 3. Mayorazgo de Pizarro-Perero}

- Escritura de compra-venta de varias casas sitas en la villa de Cáceres, pertenecientes a la agregación de Perero. Otorgada por Manuel Getino Campomanes, procurador de D. Alvaro Muñoz de Teruel y en nombre de su hijo, D. Diego Muñoz de Teruel Perero, a favor de D. Bernardo Fernández Grajar (Cáceres, 27-IX-1797). 
Carp. 4. Venta de bienes pertenecientes a los mayorazgos del duque de Abrantes.

- Copia simple de la real facultad concedida al Duque de Abrantes en 22-VII-1814 para vender algunas fincas de sus mayorazgos, hasta una valor de 2.003 .906 reales de vellón, con destino al pago de acreedores y a la reparación de fincas.

-Adjunta, en el mismo cuadernillo, copia simple del poder otorgado en 19-V-1817 por el Duque de Abrantes a favor de D. José Rodríguez Hurtado, su administrador en Cáceres, para efectuar algunas de las ventas autorizadas.

Carp. 5. Mayorazgos familiares no pertenecientes al Duque de Abrantes.

- Cuentas del mayorazgo de Quiñones, pertenecientes al Conde de Fernán-Núñez.

(No va fechado, aunque la letra es del XIX).

- Copia simple del informe realizado sobre el pleito habido entre el Duque de Frías y la Condesa de Miranda sobre la propiedad del Mayorazgo de Moguer-Villanueva del Fresno.

(No consta el año exacto del informe, pero esta copia es del siglo XIX).

\section{LEGAJO 8: CAPELLANIAS, PATRONATOS Y OBRAS PIAS}

\section{Carp. 1. Capellanías y obras pias}

A) Capellanía de D. Juan Hurtado de Mendoza, fundada en la iglesia de Santa María de Almocóvar de Alcántara en 1454:

- Traslado autorizado de los documentos de fundación y de la escritura del incremento de dotación efectuado en 1953 por D. Juan de Carvajal (Madrid,1846)

B) Capellanías fundadas en la iglesia de Santiago de Cáceres, patronato del Duqjue de Abrantes y Linares:

- Cuenta anual de las rentas y gastos de la capilla mayor de Santiago (1832).

Adjunta recibos (1832-33).

- Relaciones presentadas en la Delegación Provincial de Hacienda de Cáceres sobre los bienes de las capellanías a cargo del Duque de Abrantes y Linares (1860).

Adjunta:

Relación de todas las capellanias fundadas en la villa de Cáceres, con expresión de las personas que las poseen... (Cáceres, 1753) 
C) Obra pía fundada en la iglesia de Santiago en el siglo XVII por D. García Galarza, Obispo de Coria:

- Memoria de la obra pía que fundó el Ilmo. Sr. D. García Galarza (1832-1840).

- Traslado autorizado de la escritura de compra-venta e imposición de censo otorgada en 1755 entre el Conde de la Oliva, D. Fernando José Galarza, y D $\mathbf{a}$ Juana de Figueroa (Cáceres, 1842).

- Escritura de permuta de varias casas, otorgada entre D. Francisco León, cura ecónomo de la iglesia parroquial de Santiago de Cáceres, y D. Nicolás Roldán, administrador de las rentas del Colegio-Seminario de Coria, antes de la obra pía de D. García Galarza, ante D. Diego Ladrón de Guevara (Cáceres, 30-X-1847).

D) Obra pía de D. Andrés del Hierro Ojalvo, fundada en la iglesia de Santiago en el siglo XVIII:

- Escritura de compra-venta de un censo, otorgada por Blas Jiménez a favor de la obra pía de D. Andrés del Hierro Ojalvo ante D. Juan de la Riva Sánchez (Cáceres, 5-III-1842).

Carp. 2. Libro de caja de la capilla mayor de la iglesia de Santiago, patronato del Duque de Abrantes, formado por el administrador D. José Rodríguez Hurtado (1814-40).

296 fols.; $30 \times 20 \mathrm{~cm}$. Enc. Pergamino.

Carp. 3. Libro-memoria de las obras pías establecidas en la iglesia de Santiago de Cáceres, patronato del Duque de Abrantes y Linares (1840-71).

186 fols (181 útiles); $30 \times 20 \mathrm{~cm}$. Enc. Holanda piel.

Cap. 4. Inventario de las alhajas y efectos existentes en la capilla mayor de Santiago, propia del Duque de Abrantes y de Linares, formado con asistencia e intervención de los señores $D^{a}$ Dominica Baräibar de Lanuza y D. Sebastián Godino, administradora que ba sido la primera y administrador que es el segundo con carácter de interino... (Cáceres, 30-VI-1876).

\section{LEGAJO 9: PLEITOS}

\section{Carp. 1. Pleito entre la villa de Montánchez y el Duque de Abrantes (1793).}

- Testimonio del pleito sostenido entre la villa de Montánchez y el Duque de Abrantes, como señor de Valdefuentes, sobre la pertenencia del ganado mostrenco que se hallare fuera de los límites permitidos (Valdefuentes, 17-XIII-1793). 
El interés de este pleito radica, sobre todo, en que transcribe íntegros los siguientes documentos:

- Carta de poder otorgada por Felipe II a favor de su hermana, $\mathrm{D}^{\mathbf{a}}$ Juana de Portugal, para la venta de bienes pertenecientes a las órdenes militares (Valladolid,1555).

- Tres bulas papales autorizando la enajenación de bienes de las órdenes militares, expedidas por Julio III, Clemente VII y Pablo III respectivamente.

- Escritura de consentimiento del Comendador de Bastimentos de la Mesa Maestral de la Orden de Santiago con su ejido y dehesa boyal, a favor de D. Alvaro de Sande y su esposa, $D^{\mathbf{a}}$ Ana de Gúzmán (1588).

\section{Carp. 2. Pleito sostenido entre el Marqués de la Isla y el Duque} de Abrantes sobre el heredamiento de Aldehuela (1806-1847).

- Razón dada por Francisco de Rivera Varela de las fanegas de tierra pertenecientes al Duque de Abrantes, como poseedor del mayorazgo de los Carvajal (Cáceres, 5-IX-1806).

-Adjunta croquis.

- Dictamen emitido por D. Jacinto Hurtado sobre el deslinde y aprovechamiento de la dehesa de Aldehuela (Cáceres, 31-VII-1818).

- Razón de las tierras pertenecientes al Duque de Abrantes en el Término de Aldehuela, según las escrituras de compra (Madrid, 21-VI-1847).

- Razón de las tierras pertenecientes al Duque de Abrantes y de Linares en el heredamiento del Aldehuela, según medida prácticada por Pedro Lombas, agrimensor, el año 1773 ante el escribano Andrés Chamizo (Madrid, 23-VI-1847).

- Traslado autorizado del testimonio de apeo y deslinde efectuado en 1773 en el heredamiento de Aldehuela entre los distintos partícipes (Cáceres, 28-IX-1847).

Carp. 3. Traslado autorizado de la documentación del litigio habido entre el gremio del alfareros de Cáceres y el Duque de Abrantes sobre el arrendamiento de la dehesa de la Mejostilla. Expedido por D. Juan de la Riva Sánchez, escribano público (Cáceres, 24-II 1827).

Carp. 4. Documentación sobre el pleito seguido contra D. José Rodríguez Hurtado, administrador cesado de la hacienda del Duque de Abrantes en Cáceres (1825-30) (4 documentos) 
Carp. 5. Dictamen emitido por D. Jacinto Hurtado en el pleito seguido entre el Duque de Abrantes y otros propietarios de la dehesa "La Corchuela" sobre la cabida de cabezas de ganado (Cáceres, 1818).

Carp. 6. Autos del pleito seguido contra D. Juan Isidoro Magariño por impago del arriendo de una cerca propiedad del Duque de Abrantes, sita en el término de Torremocha (Torremocha, 1837-38).

Carp. 7. Papeles del pleito seguido por el Colegio de Humanidades de Cáceres contra el Duque de Abrantes por impago de los réditos correspondientes al año 1829 , rendidos por el préstamo recibido por su padre de D. Vicente Marrón el año 1791 (Cáceres, 1839).

(16 documentos).

\section{LEGAJO 10: RELACIONES Y CUENTAS}

\section{Carp. 1. Cuentas}

- Apuntes sobre las rentas de las dehesas del Duque de Abrantes en Cáceres (1825-30).

- Extracto de las cuentas de la administración de la hacienda del Duque de Abrantes (1826).

- Cuenta y relación jurada presentada por D. Jose López Cuervo, apoderado del Duque de Abrantes (Julio 1827-Agosto 1828).

- Haberes de la administración del Duque de Abrantes (1828-30).

- Apuntes para las cuentas de la administración (1823-32).

-Incluye los gastos extraordinarios efectuados dsde el 1 de Marzo de 1829.

- Cuentas de gastos por diferentes conceptos (1831).

- Estado y cuentas de la administración del Duque de Abrantes (Julio 1830- Junio 1831).

- Cuentas de la administración de rentas de la hacienda de Cáceres (1830-31; 1836-37; 1840-41). ( 4 cuadernillos).

- Relaciones mensuales del vencimiento de las rentas del Duque de Abrantes en Cáceres (1868-75). (8 cuadernillos).

- Cuentas de las casas pertenecientes al Duque de Abrantes, vendidas entre 1815 y 1819.

Carp. 2. Libro general de entradas y salidas de frutos (1840-54). Sin foliación; $31 X 21 \mathrm{~cm}$. Enc. Pergamino. 


\section{Carp. 3. Informes.}

- Razón de los vecinos que existen en la villa de Valdelafuente en el presente año de 1826, que han pagado el diezmo y primicias de trigo, centeno y avena.

- Notas para el informe solicitado por el Duque de Abrantes a sus arrendatarios (1828-30).

\section{LEGAJO 11:}

-DOCUMENTOS PROCEDENTES DE OTRAS HACIENDAS DEL DUQUE DE ABRANTES.

- DOCUMENTOS DE LA DIPUTACION PROVINCIAL DE CACERES.

\section{Carp. 1. Hacienda de Baza.}

- Cuentas reservadas (1823-28).

Carp. 2. Capellanías y fundaciones a cargo de la casa de Abrantes.

- Libro de caja de D. Santos García, cura propio de la villa de Hormigón (Toledo) (1751-58).

153 fols.; 21X15 cm. Enc. Pergamino.

(En los folios números 17, 21 y 129 aparecen las notas referentes a las cargas de la capellanía del Duque de Abrantes).

Carp. 3. Libro de caja y asiento de soldadas de los criados y otras cosas pertenecientes a la labor de casa (Torrijos, 1792-1813). 120 fols. útiles; $20 \mathrm{X} 15 \mathrm{~cm}$. Enc. Pergamino.

(El nombre del lugar, Torrijos, aparece por primera vez en el folio número 59).

(Se trata de un libro de cuentas en el que aparecen como firmantes dos personas distintas, los señores Amescua y Roa, lo que podría indicar que administraban una hacienda ajena, quizás propia del Duque de Abrantes, señor de varias villas cercanas a Torrijos, localidad, por otra parte, vinculada desde antiguo a varias ramas de su familia, como fueron los Cárdenas Enríquez y los Lancáster).

\section{Carp. 4. Documentos pertenecientes a la Diputación Provincial} de Cáceres.

- Libro de entradas y salidas de caudales pertenecientes a la Excelentísima Diputación de Cáceres (Enero 1845-Frebrero 1846)

Sin foliación; $24 \mathrm{X} 30 \mathrm{~cm}$. Enc. Holanda piel

(Todas las partidas aparecen firmadas por D. Tomás Leandro Lanuza, que ejerció también como funcionario de la Diputación). 


\section{B.2. DOCUMENTACION PROFESIONAL DE D. JOSE M TRUJILLO CABEZAS}

(Legs. 12-16)

HABILITACION DEL CLERO DE LA PROVINCIA DE CACERES

(Legs. 12-15)

\section{LEGAJO 12: PRESUPUESTOS}

Carp. 1. Documentos sobre presupuestos de 1862 (I) (Unica).

- Primer semestre (enero-junio).

\section{LEGAJO 13: PRESUPUESTOS}

Carp. 1. Presupuestos de 1862 (II) (Unica).

- Segundo semestre (julio-diciembre).

\section{LEGAJO 14:}

\section{-RELACIONES CON LAS DIOCESIS}

\section{COBRAS PIAS}

Carp. 1. Oficios y correspondencia diocesanos (1860-72).

- Obispado de Avila (1868).

- " " Ciudad Rodrigo (1868-71)

- " "Coria $(1860 ; 1867-69 ; 1871-72)$

- “ “ Plasencia $(1860 ; 1867-71)$.

- Arzobispado de Toledo (1868).

- Obispado-Priorato de San Marcos de León (1867-70).

Carp. 2. Obras Pías.

- Documentos relativos a la obra pía fundada en Casar de Cáceres por Da Isabel García de la Vega (1867-68).

- Obra pía fundada en Plasencia por D. José de Villanueva (1862-73).

\section{LEGAJO 15: CUENTAS Y CORRESPONDENCIA}

Carp. 1. Correspondencia y cuentas de administración y pagaduria de distritos (1860-75).

- Distrito de Alcántara

- " " " Arroyo del Puerco.

- " " " Brozas.

- “ “ Cáceres.

- “ “ Ceclavín. 


$$
\begin{aligned}
& \text { - " " Coria. } \\
& \text { - “ " " La Cumbre. } \\
& \text { - " " "Garrovillas. } \\
& \text { - “ " " Gata. } \\
& \text { - “ “ Granadilla } \\
& \text { - “ " " Guadalupe. } \\
& \text { - “ " Jaraicejo. } \\
& \text { - “ “ Jarandilla. } \\
& \text { - “ “ Montánchez. } \\
& \text { - “ " Montehermoso. } \\
& \text { - “ “ Navalmoral de la Mata. } \\
& \text { - “ " Plasencia. } \\
& \text { - “ “ Trujillo. } \\
& \text { - “ " " Valencia de Alcántara. } \\
& \text { - " " " Valverde del Fresno. } \\
& \text { - “ " Zorita. }
\end{aligned}
$$

Carp. 2. Correspondencia del Clero (1852-72).

- Adjunta algunos documentos parroquiales.

- VOCAIIA DE LA JUNTA DE INSTRUCCION PUBLICA DE LA PROVINCIA DE CACERES

- APODERAMIENTO DE AYUNTAMIENTOS

- OTROS (Leg.16)

\section{LEGAJO 16:}

Carp. 1. Vocalía de la Junta de Instrucción Pública de la provincia de Cáceres.

- Papeles referentes al Instituto de Primera Enseñanza de Cáceres (1829-45).

- Documentos relativos a diversos establecimientos escolares (1856-85).

Carp. 2. Apoderamiento del Ayuntamiento de Alcollarin (1869-74).

Carp. 3. Apoderamiento del Ayuntamiento de Garciaz (1872-73).

Carp. 4. Apoderamiento del Ayuntamiento de Zorita (1869-73).

Carp. 5. Varios.

- Correspondencia sobre asuntos bursátiles (1871-75).

- "Curriculum vitae" de D. José Ma Trujillo Cabezas. 


\section{B.3. DOCUMENTACION PROFESIONAL DE D. TOMAS TRUJILLO LANUZA \\ (Legs.17-20)}

\section{PROCURADURIA DE TRIBUNAIES}

\section{LEGAJO 17: LIBROS DE CARGO Y DATA DEL PROCURADOR D. LORENZO Ma GALLARDO (1859-62)}

Carp. 1 Libro correspondiente al año 1859. 102 fols.; 29X21 cm. Enc. Holanda piel.

Carp. 2. Año 1860 . 98 fols; 30X21 cm. Enc. Holanda piel.

Carp. 3. Año' 1861. 102 fols.; $30 X 21 \mathrm{~cm}$. Enc. Tela.

Carp. 4. Año 1862. 104 fols.; $31 \mathrm{X} 21 \mathrm{~cm}$. Enc. Tela.

\section{LEGAJO 18: LIBROS DE CARGO Y DATA DEL PROCURADOR}

D. LORENZO Ma GALLARDO (1864-72)

Carp. 1. Libro correspondiente al año 1864. 103 fols.; $31 \times 21 \mathrm{~cm}$. Enc. Tela.

Carp. 2. Año 1866. 100 fols.; $31 X 21 \mathrm{~cm}$. Enc. Tela.

Carp. 3. Año 1870 . 102 fols.; 33 X22 cm. Enc. Tela.

Carp. 4. Año 1872 . 101 fols.; $30 X 21 \mathrm{~cm}$. Enc. Tela.

LEGAJO 19: LIBROS DE ACEPTACION DE PODERES, CONOCIMIENTOS, NOTIFICACIONES Y RELACION DE CAUSAS.

LIBROS DE ACEPTACION DE PODERES DEL PROCURADOR D. LORENZO M G GALARDO

Carp. 1. Libro correspondiente al año 1863.

31 fols (sólo 3 útiles); $31 \times 21 \mathrm{~cm}$..

Enc. Tela. 
Carp. 2. Año 1868.

7 fols (sólo 2 útiles); 31X22 cm..

Enc. Tela.

Carp. 3. Año 1869.

7 fols (sólo 2 útiles); 31 X22 cm..

Enc. Tela:

Carp. 4. Año 1870 .

24 fols. (sólo 3 útiles); $32 X 21 \mathrm{~cm}$.

Enc. Tela.

\section{LIBROS DE CONOCIMIENTOS DEL PROCURADOR D. LORENZO} Ma GALLARDO

Carp. 5. Libro correspondiente al año 1861.

5 fols.; 29X21 cm. Enc. Tela

\section{LIBROS DE NOTIFICACIONES DEL PROCURADOR D. LORENZO} $M^{a}$ GALLARDO

Carp. 6. Libro correspondiente al año 1862.

24 fols. (13 útiles); $30 \times 21 \mathrm{~cm}$. Enc. Tela.

Carp. 7. Año 1863.

32 fols. (sólo 8 útiles); 29X21 cm.

Enc. Tela.

Carp. 8. Año 1864.

23 fols. (sólo 6 útiles); 29X21 cm.

Enc. Tela.

Carp. 9. Año 1870.

29 fols. (sólo 7 útiles); 32X21 cm.

Enc. Tela.

\section{LIBROS DE CORRESPONDENCIA DEL PROCURADOR \\ D. LORENZO Ma GALLARDO}

Carp. 10. Libro correspondiente al año 1861.

8 fols. sueltos.

Carp. 11. Año 1862.

83 fols. (sólo 11 útiles); $30 X 21 \mathrm{~cm}$.

Enc. Tela. 
Carp. 12. Año 1865

81 fols.; $31 \mathrm{X} 21 \mathrm{~cm}$. Enc. Tela.

(Contiene cuentas particulares de entradas y salidas de dinero, correspondientes al año 1887).

Carp. 13. Año 1896.

18 folios sueltos.

Carp. 14. Año 1868.

32 fols. (sólo 18 útiles); $33 \times 23 \mathrm{~cm}$.

Enc. Tela.

Carp. 15. Año 1869.

20 folios sueltos.

Carp. 16. Libro correspondiente al período 1870-74.

104 fols. (sólo 62 útiles); $33 \times 22 \mathrm{~cm}$.

Enc. Tela.

\section{LIBROS DE RELACION DE CAUSAS DEL PROCURADOR D. TOMAS TRUJILLO LANUZA.}

Carp. 17. Libro de Relación de Causas de Juicio Oral del Procurador D. Tomás Trujillo Lanuza (1884-86).

(Se trata en realidad de un cuaderno).

\section{LEGAJO 20}

\section{Carp. 1.}

- Nomenclator de la provincia de Cáceres becho con arreglos á la distribución judicial (1889).

223 folios.

- Nomenclätor de la provincia de Cáceres Impreso (s.e); (s.a), (¿Siglo XIX?).

\section{Carp. 2. Libros de la notaria de D. José Fernández Lázaro.}

- Libro indicador (1876-79). 150 folios.

- Libro indicador (1879-82) 199 folios. 


\section{DOCUMENTACION VARIA \\ (Leg. 21)}

\section{LEGAJO 21: DOCUMENTACION VARIA}

Carp. 1. Razón del cumplimiento de memorias y capellanías de la iglesia de San Juan de Buitrago (Guadalajara). (1785-90).

142 fols., 143-168 págs., 24 h.;

$21 \mathrm{X} 16 \mathrm{~cm}$. Enc. Pergamino.

(Aparecen como encargados de las memorias y capellanías dos sacerdotes distintos, D. Juan Pérez de Mendraza y el cura Silva. El último aparece también en el documento siguiente.

-Véase carpeta número 2-).

(No existe ningún indicio sobre la pertenencia o adjudicación del documento, aunque, con reservas, quizás podría afirmarse que su procedencia primitiva fue el archivo del Duque de Abrantes).

Carp. 2. Libro de caja de D. Manuel de Silva, cura propio de Loranca de Tajuña (Guadalajara). Abarca un período comprendido entre 1788 y 1811.

151 fols.; 21 X $15 \mathrm{~cm}$. Enc. Pergamino.

- Contiene además datos de 1814 (fol.73).

(Aparecen citados varios lugares de Extremadura, entre ellos Guadalupe) (3).

Carp. 3. Libro de criados y otros apuntamientos... para el uso de D. Francisco Ciudad, vecino de Logrosán (1849-99). 159 fols.; $21 X 16 \mathrm{~cm}$. Enc. Pergamino.

Carp. 4. Documentos sobre fincas rústicas del término de Cáceres.

- Relación de fincas, sitas en el término de Cáceres, propiedad de absentistas. Formada por D. Vicente Sánchez García (Cáceres, 1808).

- Relación de dehesa, heredamientos y yerbas del término de Cáceres (1822).

- Relación de aceras de la villa de Cáceres (sin fechar, siglo XIX).

(3) (Tampoco existe ningún indicio que permita averiguar como llegó el libro a manos del Sr. Trujillo. Sólo un hipotético y lejano parentesco del sacerdote Silva con el Duque de Abrantes, entre cuyos ascendientes se hallaba una rama de los Sivas, podría explicar algo el tema. Por otro lado, varios datos dispersos a lo largo del manuscrito parecen indicar que la zona de origen del cura Siva era Extremadura). 


\section{Carp. 5. Cofradias de Cáceres.}

A) Relación de los censos impuestos a favor de distintas cofradías de Cáceres ( $\sin$ fechar, siglo XIX).

(Faltan folios al principio).

B) Cofradía de Nuestra Señora de la Montaña (1860-98):

- ARCEDERILLO, Domingo, Noticia documental de la oposición becha en Cáceres al ejercicio legitimo de la autoridad episcopal.

(Copia manuscrita realizada por D. Tomás Trujillo Lanuza sobre la edición impresa en Cáceres por D. Nicolás Ma Jiménez el año 1860). Sin foliación.

- Contestación que da la Cofradía de Nuestra Señora de la Montaña de esta capital al folleto impreso a nombre de D. Domingo Arcederillo....sobre la intervención en la organización interior de aquélla y su orden económico.

(Copia manuscrita de la edición impresa en Cáceres, establecimiento de D. Nicolás Mํa Jiménez, 1861)

- Inventario de todos los bienes, acciones y derechos, ropas, ornamentos y albajas que pertenecen a la Virgen Santísima (1868-70). (Copia simple, manuscrita por D. Tomás Trujillo).

- Lista de individuos que componen la Junta Directiva de la Cofradía de la Montaña y lista de los bermanos o cofrades (Cáceres, 1893).

- Copia simple del inventario realizado en 1768 de las heredades del Castañar y demás tierrras de pan sembrar, propias de Nuestra Señora de la Montaña... (1898).

\section{Carp. 6. Documentos procedentes del Ayuntamiento de Alcuéscar.}

- Diligencias formadas de oficio sobre la construcción de un cementerio (Alcuéscar, 8-IX-1807).

- Libro de Acuerdos Municipales. Comprenden los siguientes períodos $1808-9 ; 1811-13 ; 1815-16 ; 1818-19 ; 1824 ; 1826-27$. 